\title{
Existence of Solutions to Generalized Vector Quasi-equilibrium Problems with Set-Valued Mappings
}

\author{
Yali Zhao*, Hong Lu and Chao Wang \\ College of Mathematics and Physics, Bohai University, Jinzhon, Liaoning 121013, China \\ ${ }^{*}$ Corresponding author
}

\begin{abstract}
In this paper, we introduce and study a class of generalized vector quasi-equilibrium problems, which includes generalized vector quasi-variational-like inequality problems, generalized vector equilibrium problems, generalized vector variational inequality problems as special cases. We use the maximal element theorem with an escaping sequence to prove the existence results of solutions for the class of generalized vector quasi-equilibrium problems without any monotonicity conditions in the setting of locally convex topological vector space. The results presented here improve and extend the corresponding results in this area.
\end{abstract}

Keywords-generalized vector quasi-equilibrium problem; maximal element theorem; upper semicontinuity; diagonal convexity; escaping sequence

\section{INTRODUCTION}

Let $X$ be a nonempty subset of a topological vector space, $E$ and $F: X \times X \rightarrow R$ be a real valued bifunction such that $F(x, x) \geq 0$ for all $x \in X$. Then the scalar equilibrium problem(in short,EP) is to find $\bar{y} \in X$ such that $F(\bar{x}, y) \geq 0$ for all $x \in X$

If $Z$ is a t.v.s. with order cone $C$; that is a closed convex pointed cone, and $F: X \times X \rightarrow Z$, then the equilibrium problem(EP) can be generalized in the following ways: Find $\bar{y} \in X$ such that $F(x, \bar{y}) \in C$ for all $x \in X$; or $F(x, \bar{y}) \notin-\operatorname{int} C$ for all $x \in X$

In these cases, (EP)are called vector equilibrium problem. These problems contains vector optimization, vector variational inequality problem and vector Nash equilibrium problem as special cases, see for example[2,3] and references therein.

Recently, Peng and Rong [4], Ahmad and Irfan [5] and Xiao et al.[6] proved some existence theorems of solutions to a class of generalized nonlinear variational inequalities. Based on these works, Gao and Feng [7] considered a class of generalized vector quasi-variational-like inequality problems and utilize the maximal element theorem with an sequence to prove the existence of its solutions in the setting of locally convex topological vector space(in short, locally convex spaces). Inspired and motivated by the above research, in this paper, we introduce a new class of generalized vector quasi- equilirium problems and follow the idea of [7], we obtain the existence results of its solutions in the setting of locally convex space, which are very interesting and improve and extend the corresponding results of [4-7].

\section{PRELIMINARIES}

Let $Z$ be a locally convex space and $X$ be a nonempty convex subset of a hausdorff topological vector space $E$ (in short, t.v.s.). We donote by $L(E, Z)$ the space of all continuous linear operators from $E$ into $Z$ and by $\langle l, x\rangle$ the evaluation of $l \in L(E, Z)$ at $x \in E$. Let $L(E, Z)$ be a space equipped with $\sigma-$ topology. By the corollary of schaefer(see page 80 in [8]), $L(E, Z)$ becomes a locally convex space. Let int $S$ and $\operatorname{coS}$ denote the interior and convex hull of a set $S$, respectively, $C: X \rightarrow 2^{Z}$ be a set-valued mapping such that int $C(x) \neq \varnothing$ for each $x \in X$, and $\eta: X \times X \rightarrow E$ be a vector-valued mapping. Let $F: L(E, Z) \times X \times X \rightarrow 2^{Z}, T: X \rightarrow 2^{L(E, Z)}, D: X \rightarrow 2^{X}$, $H: X \times X \rightarrow 2^{Z}$ be four set-valued mappings. We consider the following generalized vector quasi-equilibrium problem(in short, GVQEP):Find $\bar{x} \in X$ such that $\bar{x} \in D(\bar{x})$ and for all $y \in D(\bar{x})$, there exists $\bar{s} \in T(\bar{x})$ satistfying

$$
F(\bar{s}, y, \bar{x})+H(\bar{x}, y) \nsubseteq-\operatorname{int} C(\bar{x})
$$

The following problems are special cases of GVQEP.

(i)For all $x \in X$,if $D(x)=X$, then (2.1) redues to finding $\bar{X} \in X$, such that there exists $\bar{S} \in T(\bar{X})$ satisfying

$$
F(\bar{s}, y, \bar{x})+H(\bar{x}, y) \nsubseteq-\operatorname{int} C(\bar{x}), \forall y \in X
$$

which is a new generalized vector equilibrium problem. 
(ii)if $F(\bar{s}, y, \bar{x})=\langle A \bar{s}, \eta(y, \bar{x})\rangle$, where $A: L(E, Z) \rightarrow 2^{L(E, Z)}$ is a set-valued mapping, then (2.1) reduces to finding $x \in X$ such that $\bar{x} \in D(\bar{x})$ and for all $y \in D(\bar{x})$, there exists $\bar{S} \in T(\bar{x})$ satistfying

$$
\langle A \bar{s}, \eta(y, \bar{x})\rangle+h(\bar{x}, y) \nsubseteq-\operatorname{int} C(\bar{x})
$$

which has been studied by Gao and Feng[7].

(iii)If $A=I$ is an identity mapping on $L(E, Z)$ and $H \equiv 0$, then (2.3) reduces to finding $\bar{x} \in X$ such that $\bar{x} \in D(\bar{x})$ and for all $y \in D(\bar{x})$, there exists $\bar{y} \in T(\bar{x})$ satisfying

$$
\langle\bar{v}, \eta(y, \bar{x})\rangle \notin-\operatorname{int} C(\bar{x})
$$

which has been studied by Peng and Rong [4].

In order to prove our main results, we need the following definitions and lemmas.

Let $X$ be a topological space. A subset of $X$ is said to be compactly open(respectively, compactly closed) in $X$ if for any nonempty compact subset $K$ of $X, S \cap K$ is open(respectively, closed) in $S$. Let $Y$ be a topological space and $T: X \rightarrow 2^{Y}$ be a set-valued mapping, then $T$ is said to be open valued if the set $T(x)$ is open in $X$ for all $x \in X . T$ is said to have open lower section if $T^{-1}$ is open valued, i.e., the set $T^{-1}(y)=\{x \in X: y \in T(x)\}$ is open in $X$ for all $y \in Y$.T is said to be compactly open valued if the set $T(x)$ is compactly open in $X$ for all $x \in X$, and $T$ is said to have compactly open lower section if $T^{-1}$ is compactly open valued. Clearly, each open-valued(respectively, closed-valued) mapping $T: X \rightarrow 2^{Y}$ is compactly openvalued(respectively, compactly closed-valued). $T$ is said to be upper semicontinuous, if for any $x_{0} \in X$ and for each open set $U$ in $Y$ containing $T\left(x_{0}\right)$, there is a neighborhood $V$ of $x_{0}$ in $X$ such that $T(x) \subseteq U$ for all $x \in V ; T$ is said to be closed if the set $\{(x, y) \in X \times Y: y \in T(x)\}$ is closed in $X \times Y$.

Definition 2.1[6] Let $K$ be a convex subset of a t.v.s. $E$ and $Z$ be t.v.s. . Let $C: K \rightarrow 2^{Z}$ be a set-valued mapping. Assume given any finite subset $\Lambda=\left\{x_{1}, x_{2}, \cdots x_{n}\right\}$ of $X$, any $x=\sum_{i=1}^{n} \alpha_{i} x_{i}$ with $\alpha_{i} \geq 0$ for $i=1,2 \cdots, n$, and $\sum_{i=1}^{n} \alpha_{i}=1 \cdot$ Then (i) a single-valued mapping $f: K \times K \rightarrow Z$ is said to be vector 0-diagonally convex in the second argument if $\sum_{i=1}^{n} \alpha_{i} f\left(x, x_{i}\right) \notin-\operatorname{int} C(x)$;

(ii) a set-valued mapping $f: K \times K \rightarrow 2^{Z}$ is said to be generalized vector 0-diagonally convex in the second argument if $\sum_{i=1}^{n} \alpha_{i} f\left(x, x_{i}\right) \nsubseteq-\operatorname{int} C(x)$.

Lemma 2.1[9] Let $X$ and $Y$ be two topological spaces. If $T: X \rightarrow 2^{Y}$ is an upper semicontinuous set-valued mapping with closed values, then $T$ is closed.

Lemma2.2[10]Let $X$ and $Y$ be two topological spaces, and $T: X \rightarrow 2^{Y}$ be an upper semicontinuous set-valued mapping with compact values. Suppose that $\left\{x_{\alpha}\right\}$ is a net in $X$ such that $x_{\alpha} \rightarrow x_{0}$, if $y_{\alpha} \in T\left(x_{\alpha}\right)$ for each $\alpha$, then there is a $y_{0} \in T\left(x_{0}\right)$ and a subset $y_{\beta}$ of $y_{\alpha}$ such that $y_{\beta} \rightarrow y_{0}$.

Lemma2.3[11]Let $X$ and $Y$ be two topological spaces. Suppose that $T: X \rightarrow 2^{Y}$ is a set-valued mapping have open lower section. The set-valued mapping $F: X \rightarrow 2^{Y}$ defined by that for each $x \in X, F(x)=\operatorname{coT}(x)$ has open lower section.

Definition2.2[12]Let $E$ be a topological space and $X$ be a subset of $E$ such that $X=\bigcup_{n=1}^{\infty} X_{n}$, where $\left\{X_{n}\right\}_{n=1}^{\infty}$ is an increasing(in the sense that $X_{n} \subseteq X_{n+1}$ )sequence of nonempty compact sets. A sequence $\left\{X_{n}\right\}_{n=1}^{\infty}$ in $X$ is said to be an escaping sequence from $X$ (relative to $\left\{X_{n}\right\}_{n=1}^{\infty}$ ) if for each $n=1,2, \cdots$, there exists $m \geq 0$ such that $x_{k} \notin X_{k}$ for all $k \geq m$.

Lemma2.4[12]Let $E$ be a topological vector space and $X$ be a subset of $E$ such that $X=\bigcup_{n=1}^{\infty} X_{n}$, where $\left\{X_{n}\right\}_{n=1}^{\infty}$ is increasing sequence of nonempty compact sets of $X$. Assume that the set-valued mapping $S: X \rightarrow 2^{X}$ satisfies the following conditions:

(i)For each $x \in X, S^{-1}(x) \cap X_{n}$ is open in $X_{n}$ for all $n=1,2, \cdots$;

(ii)For each $x \in X, x \notin \operatorname{coS}(x)$;

(iii)For each sequence $\left\{X_{n}\right\}_{n=1}^{\infty}$ in $X$ with $x_{n} \in X_{n}$ for all $n=1,2, \cdots$, which is escaping from $X$ relative to 
$\left\{X_{n}\right\}_{n=1}^{\infty}$, there exists $n \in N$ and $y_{n} \in X_{n}$ such that $y_{n} \in S\left(x_{n}\right) \cap X_{n}$. Then there exists an $\bar{X} \in X$ such that $S(\bar{x})=\varnothing$.

\section{EXISTENCE RESULTS}

In this section, we prove some existence results of solutions for generalized vector quasi-equilibrium problem without any monotonicity conditions in the setting of locally convex topological vector space.

Theorem3.1 Let $E$ be a Hausdorff topological vector space, $X$ be a subset of $E$ such that $X=\bigcup_{n=1}^{\infty} X_{n}$, where $\left\{X_{n}\right\}_{n=1}^{\infty}$ is an increasing sequence of nonempty, compact and convex subset of $X$, and $Z$ be a locally convex space. Let $L(E, Z)$ be equipped with $\sigma-$ topology . Let $D: X \rightarrow 2^{X}$ be a set-valued mapping with nonempty convex value and compactly open lower section, the set $W=\{x \in X: x \in D(x)\}$ be closed, $C: X \rightarrow 2^{X}$ be a setvalued mapping such that $C(x)$ is a closed pointed and convex cone with int $C(x) \neq \varnothing$ for all $x \in X$, and the setvalued mapping $M=Z \backslash\{-\operatorname{int} C(x)\}$ be upper semicontinuous on $X$. Let $T: X \rightarrow 2^{L(E, Z)}$ be upper semicontinuous on $X$ with compact values and $H: X \times X \rightarrow 2^{Z}$ be generalized vector 0-diagonally convex in the second argument. Let $F: L(E, Z) \times X \times X \rightarrow 2^{Z}$ be affine in the second argument with $F(s, x, x) \subseteq C(x)$ for all $(s, x) \in L(E, Z) \times X \quad$. For each $y \in X$, assume that $F(\cdot, y, \cdot)+H(\cdot, y): L(E, Z) \times X \times X \rightarrow 2^{Z}$ is an upper semicontinuous set-valued mapping with compact value. Suppose that the following condition holds:

(C)for each sequence $\left\{X_{n}\right\}_{n=1}^{\infty}$ in $X$ with $x_{n} \in X_{n}$ for all $n=1,2, \cdots$, which is escaping from $X$ relative to $\left\{X_{n}\right\}_{n=1}^{\infty}$, there exist $m \in N$ and $z_{m} \in D\left(x_{m}\right) \cap x_{m}$ such that for all $s_{m} \in T\left(x_{m}\right), F\left(s_{m}, z_{m}, x_{m}\right)+H\left(x_{m}, z_{m}\right) \subseteq-\operatorname{int} C\left(x_{m}\right)$.

Then GVQEP has a solution.

Proof. Define a set-valued mapping $P: X \rightarrow 2^{X}$ by

$$
P(x)=\{y \in X: F(s, y, x)+H(x, y) \subseteq-\operatorname{int} C(x), \forall s \in T(x)\},
$$
$\forall x \in X$.We first prove that $x \notin \operatorname{coP}(x)$ for all $x \in X$. To see this, by way of contradiction, assume that there exists some point $\bar{X} \in X$ such that $\bar{X} \in \operatorname{CoP}(\bar{x})$. Then there exist finite points $y_{1}, y_{2}, \cdots y_{n}$ in $P(\bar{x})$, and $\alpha_{j} \geq 0$ with $\sum_{j=1}^{n} \alpha_{j}=1$ such that $\bar{x}=\sum_{j=1}^{n} \alpha_{j} y_{j}$. That is, $F\left(s, y_{j}, \bar{x}\right)+H\left(\bar{x}, y_{j}\right) \subseteq-\operatorname{int} C(\bar{x}), \forall s \in T(\bar{x}), i=1,2, \cdots n \quad$ Since $\operatorname{int} C(\bar{x})$ is a convex set and $F$ is affine in the second argument with $F(s, x, x) \subseteq C(x)$ for all $(s, x) \in L(E, Z) \times X$, we have $F(s, \bar{x}, \bar{x})+\sum_{j=1}^{n} \alpha_{j} H\left(\bar{x}, y_{j}\right) \subseteq-\operatorname{int} C(\bar{x})$ implying that $\begin{aligned} \sum_{j=1}^{n} \alpha_{j} H\left(\bar{x}, y_{j}\right) & \subseteq-\operatorname{int} C(\bar{x})-F(s, \bar{x}, \bar{x}) \\ & \subseteq-\operatorname{int} C(\bar{x})-C(\bar{x}) \\ & \subseteq-\operatorname{int} C(\bar{x}) .\end{aligned}$

which contradicts the fact that $H$ is generalized vector 0 diagonal convex in the second argument. Therefore, $x \notin \operatorname{coP}(x)$ for all $x \in X$. We also define a set-valued mapping $G: X \rightarrow 2^{X}$ by $G(x)=\left\{\begin{array}{c}D(x) \cap \operatorname{coP}(x), x \in W, \\ D(x), \quad x \in X \backslash W .\end{array}\right.$

Then, for each $x \in X, G(x)$ is convex. Suppose that there exists $\bar{x} \in X$ such that $\bar{x} \in G(\bar{x})$. If $\bar{x} \in W$, then $\bar{x} \in D(\bar{x}) \cap \operatorname{coP}(x)$, which contradicts $x \notin \operatorname{coP}(x)$ for all $x \in X$. If $\bar{x} \notin W$, then $G(\bar{x})=D(\bar{x})$ which implies $\bar{x} \in D(\bar{x}), \quad$ a contradiction. Hence $x \notin G(x)=\operatorname{coG}(x), \forall x \in X$, and the condition (ii) of Lemma 2.4 is satisfied. Next, we prove that the set $P^{-1}(y)=\{x \in X: F(s, y, x)+H(x, y) \subseteq-\operatorname{int} C(x), \forall s \in T(x)\}$ is open for all $y \in X$. That is, $P$ has open lower section in $X$. Consider the set $\left(P^{-1}(y)\right)^{c}=\left\{\begin{array}{l}x \in X:[F(s, y, x)+H(x, y)] \cap Z \backslash(-\operatorname{int} C(x)) \neq \varnothing, \\ \exists s \in T(x)\end{array}\right\}$ which is the complement of $\left(P^{-1}(y)\right)$. We only need to prove that $\left(P^{-1}(y)\right)^{c}$ is closed for all $y \in X$. Let $\left\{x_{\alpha}\right\}$ be a net in $\left(P^{-1}(y)\right)^{c}$ such that $x_{\alpha} \rightarrow x^{*}$. Then there exists an $s_{\alpha} \in T\left(x_{\alpha}\right)$ such that

$$
\left[F\left(s_{\alpha}, y, x_{\alpha}\right)+H\left(x_{\alpha}, y\right)\right] \cap Z \backslash\left(-\operatorname{int} C\left(x_{\alpha}\right)\right) \neq \varnothing .
$$

Since $T: X \rightarrow 2^{L(E, Z)}$ is an upper semicontinuous setvalued mapping with compact values, by Lemma 2.2, $\left\{s_{\alpha}\right\}$ has a convergent subsequence with limit, say $s^{*}$, and $s^{*} \in T\left(x^{*}\right)$. Without loss of generalizity, we may assume that $s_{\alpha} \rightarrow s^{*}$. Suppose that

$$
Z_{\alpha} \in\left[F\left(s_{\alpha}, y, x_{\alpha}\right)+H\left(x_{\alpha}, y\right)\right] \cap Z \backslash\left\{-\operatorname{int} C\left(x_{\alpha}\right)\right\} .
$$

Since $F(\cdot, y)+,H(\cdot, y)$ is upper semicontinuous with compact value, by Lemma 2.2, there exists a 
$z^{*} \in F\left(s^{*}, y, x^{*}\right)+H\left(x^{*}, y\right)$ and a subsequence $\left\{z_{\beta}\right\}$ of $\left\{z_{\alpha}\right\}$ such that $z_{\beta} \rightarrow z^{*}$. On the other hand, since $Z \backslash\left\{-\operatorname{int} C\left(x_{\alpha}\right)\right\}$ is upper semicontinuous with closed values, by Lemma 2.1,we have $Z^{*} \in Z \backslash\left\{-\operatorname{int} C\left(x^{*}\right)\right\}$. Hence $\left[F\left(s^{*}, y, x^{*}\right)+H\left(x^{*}, y\right)\right] \cap Z \backslash\left\{-\operatorname{int} C\left(x^{*}\right)\right\} \neq \varnothing$. Thus, $\left(P^{-1}(y)\right)^{c}$ is closed in $X$. Therefore, $P$ has open lower section in $X$. By Lemma 2.3, $\operatorname{coP}^{-1}(y)$ is also open for all $y \in X$. Since $D^{-1}(y)$ is compact open for all $y \in X$, we have

$$
\begin{aligned}
G^{-1}(y)= & \{x \in X: y \in G(x)\} \\
= & \{x \in W: y \in[D(x) \cap \operatorname{co}(x)]\} \\
& \cap\{x \in X \backslash W: y \in D(x)\} \\
= & {\left[\left(W \cap D^{-1}(y) \cap c o P^{-1}(y)\right) \cup(X \backslash W)\right] } \\
& \cap\left[\left(W \cap D^{-1}(y) \cap \operatorname{coP}^{-1}(y)\right) \cup D^{-1}(y)\right] \\
= & \left\{\begin{array}{l}
X \cap\left[\left(D^{-1}(y) \cap c o P^{-1}(y)\right) \cup(X \backslash W)\right] \\
\cap\left[\left(W \cup D^{-1}(y)\right) \cap\left(D^{-1}(y)\right)\right]
\end{array}\right\} \\
= & {\left.\left[\left(D^{-1}(y) \cap \operatorname{coP} P^{-1}(y)\right) \cup(X \backslash W)\right] \cap D^{-1}(y)\right) } \\
= & \left(D^{-1}(y) \cap c o P^{-1}(y)\right) \cup\left((X \backslash W) \cap\left(D^{-1}(y)\right)\right) .
\end{aligned}
$$

Therefore, $G^{-1}(y)$ also has compactly open values in $X$ for all $y \in X$, the condition (i) of Lemma 2.4 is satisfied. Condition (C) implies that the condition (iii) of Lemma 2.4. It follows from Lemma2.4 that there exists an $\bar{X} \in X$ such that $G(\bar{x})=\varnothing$.

Since for each $x \in X, D(x)$ is nonempty, we have $\bar{x} \in D(\bar{x})$ such that $D(\bar{x}) \cap P(\bar{x})=\varnothing$, that is, there has an $\bar{x} \in D(\bar{x})$, and for all $y \in D(\bar{x})$, there exists an $\bar{s} \in T(\bar{x})$ satisfying $F(\bar{s}, y, \bar{x})+H(\bar{x}, y) \nsubseteq-\operatorname{int} C(\bar{x})$

This completes the proof.

Theorem3.2 Let $E, X, Z, L(E, Z), D, W, C, M, F, T$ be the same as in Theorem 3.1. For each $y \in X$, assume that $F(\cdot, y, \cdot)+H(\cdot, y): L(E, Z) \times X \times X \rightarrow 2^{Z} \quad$ is an upper semicontinuous set-valued mapping with compact values. Suppose that there exists a mapping $R: X \times X \rightarrow 2^{Z}$,

(i)There exists a $s \in T(x)$ for all $x, y \in X$, such that $R(x, y)-[F(s, y, x)+H(x, y)] \subseteq-\operatorname{int} C(x)$
(ii)For any finite set $\left\{y_{1}, y_{2} \cdots y_{n}\right\} \subseteq X$ and $\bar{x}=\sum_{j=1}^{n} \alpha_{j} y_{j}$, with $\alpha_{j} \geq 0$ and $\sum_{j=1}^{n} \alpha_{j}=1$, there is a $j \in\{1,2, \cdots n\}$ such that $R\left(\bar{x}, y_{j}\right) \nsubseteq-\operatorname{int} C(\bar{x})$;

(iii)For each sequence $\left\{X_{n}\right\}_{n=1}^{\infty}$ in $X$ with $x_{n} \in X_{n}$ for all $n=1,2, \cdots$, which is escaping from $X$ relative to $\left\{X_{n}\right\}_{n=1}^{\infty}$, there exist an $m \in N$ and $z_{m} \in D\left(x_{m}\right) \cap x_{m}$ such that

$$
F\left(s_{m}, z_{m}, x_{m}\right)+H\left(x_{m}, z_{m}\right) \subseteq-\operatorname{int} C\left(x_{m}\right), \forall s_{m} \in T\left(x_{m}\right) .
$$

Then GVQEP has a solution.

Proof. Define two set-valued mappings $P_{1}, P_{2}: X \rightarrow 2^{X}$ by $P_{1}(x)=\{y \in X: F(s, y, x)+H(x, y) \subseteq-\operatorname{int} C(x), \forall s \in T(x)\}$, $\forall x \in X ; P_{2}(x)=\{y \in X: R(x, y) \subseteq-\operatorname{int} C(x)\}, \forall x \in X$.

We first prove that $X \notin \operatorname{coP}_{2}(x)$ for all $x \in X$. For this, by way of contradiction, assume that there exists some point $\bar{X} \in X$ such that $\bar{X} \in \operatorname{CoP}_{2}(x)$. Then there exist finite points $y_{1}, y_{2}, \cdots y_{n}$ in $X$ and $\alpha_{j} \geq 0$ with $\sum_{j=1}^{n} \alpha_{j}=1$ such that $\bar{x}=\sum_{j=1}^{n} \alpha_{j} y_{j}$ and $y_{j} \in P_{2}(\bar{x})$ for all $j=1,2, \cdots n$.That is, $R\left(\bar{x}, y_{j}\right) \subseteq-\operatorname{int} C(\bar{x}), j=1,2, \cdots, n$. This contradicts the condition (ii). So, $x \notin C O P_{2}(x)$ for all $x \in X$.

The condition (i) implies that $P_{2}(x) \supseteq P_{1}(x)$ for all $x \in X$. Hence, $x \notin \operatorname{coP}_{1}(x)$ for all $x \in X$. The remainder of the proof is the same as that of Theorem3.1, so is omitted, completing the proof.

Remark3.1 Theorem 3.1 and Theorem 3.2 improve and extend the corresponding results of [4-7].

\section{ACKNOWLEDGMENT}

This work is supported by the National Natural Science Foundation of China (11371070).

\section{REFERENCES}

[1] E. Blum , and W.Oettli , "From optimization and Variational inequalities to equilibrium problem,” The Mathematics Students, 1994, 63: 123-146.

[2] M.Balaj, "Coincidence and maximal element theorems and their applications to generalized equilibrium problems and minimax inequalities,” Nonlinear AnalTMA, 2008, 68: 3962-3971.

[3] X. P. Ding, "The generalized game and the system of generalized vector quasi-equlibrium problems in locally FC-uniform spaces," Nonlinear Anal. TMA, 2008, 68: 1028-1036.

[4] J. W. Peng, and W. D.Rong, “Generalized vector quasi-variational-like inequalities without monotonicity and compactness,” J. Math. Res. Exposition, 2005, 25: 415-422. 
[5] R. Ahmad, and S. S. Irfan, "On generalized nonlinear variational-like inequalities problems,” Appl. Math. Lett., 2006, 19: 294-297.

[6] G. Xiao , Z. Q. Fan , and X. G. Qi ,"Existence results for generalized nonlinear vector variational-like inequalities with set-valued mapping,”Appl. Math. Lett, 2010, 23: 44-47.

[7] D. P. Gao , and S. Q. Feng , "Existence of solutions to generalized vector quasi-variational-like inequalities with set-variational-like inequalities with set-valued mappings," Communications in Mathematical Reseeearch, 2014, 30(1): 90-96.

[8] H. H. Schaefer, “Topological Vector Space," Graduate Texts in Mathematics. vol. 3. New York: Springer, 1980.

[9] J. P. Aubin, and I. Ekeland, “Applied Nonlinear Analysis,” New York: John Wiley and Sons, Inc., 1984.

[10] C. H. Su , and V. M.Sehgal , "Some fixed point theorems for condensing multifunctions in locally convex spaces,” Proc. Amer. Math. Soc., 1975, 50: $150-154$

[11] G. Q. Tian , and J. X.Zhou "QQuasi-variational inequalities without the concavity assumption,”J. Math. Anal. Appl., 1993, 172(1): 289-299.

[12] G. X.Yuan , Z. G. Isac, and K. K.Tan, "The study of minimax inequalities, abstract economics and applications to variational inequalities and Nash equilibraia,” Acta Appl. Math., 1998, 54: 135-166. 